\title{
Comportamento de acadêmicos de enfermagem frente aos riscos para acidente com material perfurocortante
}

\author{
Behavior of nursing students regarding the risks of accidents with sharps \\ Comportamiento de estudiantes de enfermería ante los riesgos de accidentes con objetos punzantes
}

Recebido: 27/01/2021 | Revisado: 04/02/2021 | Aceito: 09/02/2021 | Publicado: 13/02/2021

\author{
Carlos Eduardo Bezerra Monteiro \\ ORCID: https://orcid.org/0000-0003-1787-1892 \\ Universidade Federal do Amazonas, Brasil \\ E-mail: edumonteiro_123@hotmail.com \\ Francisca Moreira Dantas \\ ORCID: https://orcid.org/0000-0002-5088-5287 \\ Secretaria Municipal de Saúde de Uarini, Brasil \\ E-mail: franmdantas@hotmail.com \\ Josiane Montanho Mariño \\ ORCID: https://orcid.org/0000-0002-2876-0226 \\ Universidade Federal do Amazonas, Brasil \\ E-mail: enf_josiane@yahoo.com.br
}

\begin{abstract}
Resumo
O estudo tem por objetivo verificar o comportamento frente aos riscos para acidente com material perfurocortante em estudantes de graduação em enfermagem, de uma Instituição de Ensino Superior pública do interior do Amazonas, Brasil. A presente pesquisa é de caráter quantitativo, descritivo, transversal, tendo como público alvo os graduandos do curso de bacharel em enfermagem do Instituto de Saúde e Biotecnologia da Universidade Federal do Amazonas. A realização da coleta de dados se deu por 2 entrevistadores previamente treinados, nos meses de Julho a Agosto de 2017. A amostra foi composta por 94 estudantes, destes, 62,8\% eram do sexo feminino, média de idade de 22,5 anos, e 78,7\% eram solteiros. Constatou-se que a maioria $(89,7 \%)$ utilizavam Equipamentos de Proteção Individual nas aulas práticas e a prevalência de 5 ocorrências de acidentes com material perfurocortante. Sugere-se pesquisas adicionais para averiguar condutas tomadas em relação aos acidentes com material perfurocortante atingindo esse público.
\end{abstract}

Palavras-chave: Contenção de riscos biológicos; Riscos ocupacionais; Estudantes de enfermagem; Ensino.

\begin{abstract}
The study aims to verify the behavior regarding the risks for accidents with sharps in undergraduate nursing students, from a public Higher Education Institution in the interior of Amazonas, Brazil. This research is quantitative, descriptive, cross-sectional, with the target audience of undergraduate nursing students at the Institute of Health and Biotechnology of the Federal University of Amazonas. The data collection was carried out by 2 previously trained interviewers, from July to August 2017. The sample consisted of 94 students, of which $62.8 \%$ were female, mean age 22.5 years, and $78.7 \%$ were single. It was found that the majority $(89.7 \%)$ used Personal Protective Equipment in practical classes and the prevalence of 5 accidents with sharps. Additional research is suggested to investigate the actions taken in relation to accidents with sharps that reach this public.
\end{abstract}

Keywords: Containment of biohazards; Occupational risks; Students nursing; Teaching.

\section{Resumen}

El estudio tiene como objetivo verificar el comportamiento frente a los riesgos de accidentes con objetos punzantes en estudiantes de pregrado de enfermería, de una Institución de Educación Superior pública en el interior de Amazonas, Brasil. Esta investigación es cuantitativa, descriptiva, transversal, con el público objetivo de estudiantes de pregrado en enfermería del Instituto de Salud y Biotecnología de la Universidad Federal de Amazonas. La recolección de datos fue realizada por 2 entrevistadores previamente capacitados, de julio a agosto de 2017. La muestra estuvo conformada por 94 estudiantes, de los cuales $62,8 \%$ eran mujeres, edad media 22,5 años y 78,7\% solteros. Se encontró que la mayoría $(89,7 \%)$ utilizó Equipo de Protección Personal en las clases prácticas y la prevalencia de 5 accidentes con objetos punzantes. Se sugiere realizar investigaciones adicionales para investigar las acciones tomadas en relación a los accidentes con objetos punzantes que llegan a este público.

Palabras clave: Contención de riesgos biológicos; Riesgos laborales; Estudiantes de enfermería; Enseñanza. 


\section{Introdução}

Durante o processo de formação do estudante de enfermagem faz-se necessário a realização de aulas práticas, e tais práticas são executadas em instituições de saúde hospitalares. Essas instituições hospitalares prestam assistência à saúde no intuito de acolher, tratar e curar diversas doenças (Amaral, Scherer, \& Trindade, 2019).

Com isso, esses acadêmicos se tornam expostos por desempenharem atividades práticas semelhantes ao do profissional já formado, no intuito de desenvolver e adquirir habilidades necessárias voltadas para o cuidado com os pacientes, que os coloca em risco de acidente de diversas naturezas, dentre eles o acidente com material perfurocortante (Cardoso et al., 2019; Lima, Rocha, Lima, \& Amendola, 2016).

O manuseio com objetos perfurocortantes com alunos de graduação em enfermagem, também pode ser considerado de alto risco para a ocorrência de acidente que contenha material biológico durante a prática acadêmica (Cardoso et al., 2019).

Em alguns casos envolvendo acidentes com graduandos é possível identificar que os mesmos afirmam obterem bons conhecimentos sobre a biossegurança, porém, muitos não aplicam tais conhecimentos durante a prática, negligenciando procedimentos básicos de prevenção e consequentemente tornando-os expostos ao risco (Bastos et al., 2020; Santos, Hermes, Susin, \& Moreira, 2017).

A classe da enfermagem torna-se mais susceptível ao risco e vulnerável aos acidentes, devido ao maior contato com os pacientes. Isso ocorre devido as atribuições em realizar tarefas invasivas ou em manipular materiais perfurocortantes deixando-os mais expostos (Carvalho \& Luz, 2018).

Assim como os profissionais desempenham atendimento hospitalar, lidando diretamente com pacientes, e realizam tarefas que muitas vezes são consideradas rotineiras, os discentes também se encontram envolvidos nesse contexto. Atrelado a essas atribuições e execução de atividades, o graduando se depara com a falta de experiência, a ansiedade e o estresse, somado a isso, estão em constante aprendizagem, o que pode contribuir para o acontecimento de acidentes (Cardoso et al., 2019; Carvalho, Rocha, Gimenes, Santos, \& Valim, 2018).

$\mathrm{O}$ estudo tem por objetivo verificar o comportamento frente aos riscos para acidente com material perfurocortante em estudantes de graduação em enfermagem, de uma Instituição de Ensino Superior (IES) pública do interior do Amazonas, Brasil.

\section{Metodologia}

\section{Aspectos Éticos}

Em conformidade com a Resolução 466/12 do Conselho Nacional de Saúde, o estudo ora apresentando foi submetido ao Comitê de Ética em Pesquisa (CEP) da Universidade Federal do Amazonas (UFAM), que aprovou conforme Certificado de Apresentação para Apreciação Ética (CAAE) 67016317.6.0000.5020, resguardando todos os aspectos éticos, de acordo com os amparos legais vigentes (Brasil, 2012).

\section{Desenho do Estudo e Critérios de Inclusão e Exclusão}

Trata-se de um estudo de pesquisa de caráter quantitativo, descritivo, transversal, aninhado à pesquisa intitulada "Hepatite B e C em estudantes de enfermagem: prevalência e associação com os fatores de risco", tendo como público alvo os graduandos de todos os semestres do curso de bacharel em enfermagem do Instituto de Saúde e Biotecnologia (ISB) da UFAM, localizado no município de Coari, Amazonas, Brasil.

A amostra foi composta por 94 estudantes de graduação em enfermagem, de ambos os sexos, matriculados regularmente nos cinco semestres do curso, selecionados de forma aleatória. Estiveram excluídos da pesquisa os acadêmicos afastados de sala de aula quando o motivo da reclusão não se relacione com o evento estudado, a exemplo dos casos de licença 
maternidade e outras patologias. Foram excluídos, ainda, os alunos oriundos de povos indígenas, bem como os discentes com matrículas trancadas.

\section{Procedimentos}

A realização da coleta de dados se deu por 2 entrevistadores previamente treinados, nos meses de Julho a Agosto de 2017 no laboratório de enfermagem do ISB. Os pesquisadores envolvidos explicaram os objetivos, procedimentos e destino dos dados, bem como informaram que a participação na pesquisa consistia de forma voluntária e que os resultados seriam tratados com confidencialidade, garantindo o anonimato das informações. Nessa ocasião, os participantes foram convidados a fazer parte do presente estudo, aos que aceitaram foram direcionados para o laboratório de enfermagem sendo fornecidas todas as informações pertinentes à pesquisa, bem como sanando possíveis dúvidas que pudessem surgir, nesse mesmo local foram coletadas as informações de cada aluno em local privado. Os entrevistados participantes assinaram duas vias do Termo de Consentimento Livre e Esclarecido (TCLE).

\section{Instrumentação}

Para a coleta de dados aplicou-se um questionário composto por três blocos, entretanto, para a realização de tal estudo, utilizou-se as seguintes variáveis: sexo, idade, situação conjugal, semestre em que estava cursando, realização de aulas práticas, acidente com material perfurocortante durante as aulas práticas, e utilização dos Equipamentos de Proteção Individual (EPIs) durante as aulas práticas.

A tabulação e análise dos dados foram realizadas com o auxilio do software Statistical Package for the Social Sciences (SPSS) 20.0 for Windows.

\section{Resultados e Discussão}

Dentre os graduandos pesquisados $62,8 \%$ pertenciam ao sexo feminino e 37,2\% do sexo masculino, com faixa etária entre 16 e 39 anos, obtendo uma média de idade de 22,5 (SD=4,6) anos. Quanto à situação conjugal a maior parte $(78,7 \%)$ eram solteiros, havendo a predominância dos estudantes do $5^{\circ}$ semestre $(29,8 \%)$ na pesquisa (Tabela 1$)$. 
Tabela 1 - Caracterização dos estudantes de enfermagem do Instituto de Saúde e Biotecnologia da Universidade Federal do Amazonas, de acordo com as variáveis sociodemográficas. Coari, AM, 2017.

\begin{tabular}{|c|c|c|}
\hline \multirow{2}{*}{ Variáveis } & \multicolumn{2}{|c|}{ Estudantes de Enfermagem } \\
\hline & Frequência (n=94) & Porcentagem (\%) \\
\hline \multicolumn{3}{|l|}{ Sexo } \\
\hline Masculino & 35 & 37,2 \\
\hline Feminino & 59 & 62,8 \\
\hline \multicolumn{3}{|l|}{ Idade (anos) } \\
\hline $16-20$ & 34 & 36,2 \\
\hline $21-25$ & 43 & 45,7 \\
\hline $26-30$ & 10 & 10,6 \\
\hline $31-35$ & 4 & 4,3 \\
\hline$\geq 36$ & 3 & 3,2 \\
\hline Média de idade (Desvio Padrão) & $22,5(4,6)$ & \\
\hline \multicolumn{3}{|l|}{ Situação Conjugal } \\
\hline Solteiro & 74 & 78,7 \\
\hline Casado/Vive junto & 20 & 21,3 \\
\hline \multicolumn{3}{|l|}{ Semestre } \\
\hline $1^{\circ}$ & 26 & 27,7 \\
\hline $3^{\circ}$ & 10 & 10,6 \\
\hline $5^{\circ}$ & 28 & 29,8 \\
\hline $7^{\circ}$ & 19 & 20,2 \\
\hline $9^{\circ}$ & 11 & 11,7 \\
\hline
\end{tabular}

Fonte: Autores (2021).

Dos alunos entrevistados 58 já haviam realizado aulas práticas, 8,6\% destes já haviam se acidentado com material perfurocortante durante o período das práticas hospitalares e 10,3\% costumavam utilizar às vezes os EPIs necessários durante a realização dos procedimentos (Tabela 2).

Tabela 2 - Caracterização dos estudantes de enfermagem do Instituto de Saúde e Biotecnologia da Universidade Federal do Amazonas, de acordo com as variáveis comportamentais. Coari, AM, 2017.

\begin{tabular}{l|cc}
\hline \multirow{2}{*}{ Variáveis } & \multicolumn{2}{c}{ Estudantes de Enfermagem } \\
\cline { 2 - 3 } & Frequência & Porcentagem (\%) \\
\hline $\begin{array}{l}\text { Acidente com material perfurocortante } \\
\text { durante as aulas práticas }\end{array}$ & 5 & \\
$\quad$ Sim & 53 & 8,6 \\
Não & & \\
Utilização dos EPIs durante as aulas & 52 & 89,4 \\
práticas & 6 & 10,3 \\
$\quad$ Sempre & \\
As vezes & \multicolumn{2}{c}{} \\
\multicolumn{2}{c}{ Fonte: Autores (2021). }
\end{tabular}

A predominância do sexo feminino e o perfil jovem dos acadêmicos encontrados no estudo estão em concordância com outras investigações realizadas com estudantes de enfermagem (Cardoso et al., 2019; Oliveira \& Braga, 2016; Reza et al., 2016). Esses dados apontam para o predomínio da classe feminista nos cursos de enfermagem, principalmente por influência cultural advinda desde os primórdios da profissão. Entretanto, esse cenário vem se transformando com a crescente presença da classe masculina nos cursos de enfermagem.

Detectou-se a prevalência de discentes solteiros $(78,7 \%)$ no período da pesquisa, havendo um indicativo da relação com o perfil jovem da população em estudo, dados que coincidem com outros estudos realizados apontando a mesma realidade 
deparada (Bublitz, Guido, Kirchhof, Neves, \& Lopes, 2015; Oliveira, Nunes, \& Mourão, 2015).

Verificou-se no presente estudo a existência de acidentes com material perfurocortante, tal dado corrobora com outros estudos (Oliveira, Macedo, Morais, Tanan, \& Yarid, 2017; Santos Junior, Batista, Almeida, \& Abreu, 2015) que registram casos de acidente na mesma classe estudantil. Esse tipo de material também é destaque em acidentes ocorridos entre graduandos de enfermagem (Oliveira et al., 2017).

A utilização dos EPIs durante as aulas práticas esteve predominante na maioria, correspondendo $89,7 \%$. Tal dado entra em concordância com o encontrado no estudo de Chehuen Neto et al. (2018) com estudantes da área de saúde da Universidade Federal de Juiz de Fora (UFJF), dentre os alunos entrevistados da enfermagem todos afirmaram utilizar frequentemente os EPIs.

Mesmo identificando que a maioria sempre utilizava EPI nas aulas práticas, observou-se que alguns (10,3\%) informaram usar às vezes. Representando um dado preocupante e importante para discutir no âmbito da graduação. Tendo em vista que o EPI configura-se como uma proteção para o individuo durante a execução de determinada atribuição, garantido a minimização dos riscos e acidentes decorrentes de tal atividade (Garcez et al., 2019).

Diante dessas práticas adotadas ainda durante o processo de formação há a possibilidade de no futuro enquanto profissionais permanecerem com determinados hábitos, caracterizando um agravo posterior. Nesse contexto, um estudo realizado com profissionais e estudantes da área da saúde evidenciou que durante o momento do acidente a grande maioria (cerca de 78,0\%) não utilizava o EPI necessário, o que reforça mais ainda a importância do EPI no meio acadêmico (Santos Junior et al., 2015).

O curso de enfermagem do ISB possui em sua matriz curricular vigente a disciplina Biossegurança, entretanto, a mesma encontra-se inserida como optativa, sugerindo indícios da possibilidade de existir profissionais formados que não cursaram essa disciplina. Muito embora, tal assunto é abordado em diversas outras disciplinas de caráter teoria-prática.

Frente a esses apontamentos, faz-se necessário redobrar a atenção e reforçar as medidas de biossegurança por parte dos enfermeiros docentes, tanto nas aulas práticas quanto nas aulas teóricas, buscando diminuir os riscos e os números de acidentes que envolvam discentes da área da enfermagem (Morais et al., 2017).

\section{Considerações Finais}

No presente estudo constatou-se que a maioria dos estudantes de enfermagem utilizavam EPIs durante as aulas práticas e a existência de 5 acontecimentos de acidentes com material perfurocortante. Apesar do quantitativo pequeno, obteve a representatividade de $8,6 \%$ dos alunos que já estavam na fase de aulas práticas no ambiente hospitalar.

Em virtude de a enfermagem encontrar-se em constante contato com os pacientes, torna-se essencial que os acadêmicos sejam conscientizados da fundamental importância do uso contínuo e adequados dos EPIs. O estudo ainda sugere pesquisas adicionais no intuito de averiguar condutas tomadas em relação aos acidentes com material perfurocortante atingindo estudantes de enfermagem.

Cabe destacar como limitação do presente estudo, que os dados apresentados refletem uma realidade local, não podendo ser generalizado a nível regional, nacional e/ou internacional.

\section{Agradecimentos}

Fundação de Amparo à Pesquisa do Estado do Amazonas.

Instituto de Medicina Tropical de Coari-AM. 


\section{Referências}

Amaral, G. S., Scherer, M. D. A., \& Trindade, L. L. (2019). Contribuições e desafios do enfermeiro supervisor na formação aca dêmica de enfermagem em contexto hospitalar. Tempus Actas de Saúde Coletiva, 13(2), 23-36.

Bastos, A. P. S., Brito, M. A. R., Ferreira, J. L. R., Ferreira, J. G. O. R., Araujo, V. S. C., Val, H. M. C., Mouta, A. A. N., Campelo, Y. D. M., Jesus, J. R., \& Beltrão, R. P. L. (2020). Equipamentos de proteção individual e a adesão do conhecimento dos profissionais e acadêmicos: revisão integrativa. Revista Eletrônica Acervo Saúde, (53), e3764.

Brasil (2012). Conselho Nacional de Saúde. Resolução n 466, 2012. Diretrizes e Normas regulamentadoras de pesquisa envolvendo seres humanos. Brasília. Seção 1 p. 59.

Bublitz, S., Guido, L. A., Kirchhof, R. S., Neves, E. T., \& Lopes, L. F. D. (2015). Perfil sociodemográfico e acadêmico de discentes de enfermagem de quatro instituições brasileiras. Revista Gaúcha de Enfermagem, 36(1), 77-83.

Cardoso, N. Q., Ream, P. S. F., Souza, C. L., Salgado, T. A., Galdino Júnior, H., \& Tipple, A. F. V. (2019). Acidente com material biológico sob a ótica dos estudantes de enfermagem: reflexões para o ensino. Enferm. foco, 10(3), 2-8.

Carvalho, D. C., Rocha, J. C., Gimenes, M. C. A., Santos, E. C., \& Valim, M. D. (2018). Acidentes de trabalho com material biológico na equipe de enfermagem de um hospital do Centro-Oeste brasileiro. Escola Anna Nery, 22(1), e20170140.

Carvalho, T. S., \& Luz, R. A. (2018). Acidentes biológicos com profissionais da área da saúde no Brasil: uma revisão da literatura. Arquivos Médicos dos Hospitais e da Faculdade de Ciências Médicas da Santa Casa de São Paulo, 63(1), 31-36.

Chehuen Neto, J. A., Lima, M. G., Santos, J. L. C. T., Costa, L. A., Estevanin, G. M., Freire, M. R., \& Ferreira, R. E. (2018). Conhecimento e adesão às práticas de biossegurança entre estudantes da área da saúde. Brazilian Journal of Surgery and Clinical Research, 21(2), 82-87.

Garcez, G. O., Tavares, L. M., Bernardes, L. L. P., Dutra, P. V. R., Valle, P. S., \& Álvares, A. C. M. (2019). Uso de EPI's pelos profissionais de saúde da atenção básica de um município de Goiás. Revista de Divulgação Científica Sena Aires, 8(4), 418-426.

Lima, E. A. G., Rocha, I. B., Lima, D., \&Amendola, F. (2016). Revisão Integrativa sobre acidente de trabalho com pérfuro cortante em profissionais de enfermagem. Revista Saúde, 10(1-2), 71-86.

Morais, R. L. G. L., Tanan, M. S., Oliveira, J. S., Macedo, M. P., Nery, A. A., \& Matos Filho, S. A. (2017). Conhecimentos e condutas de biossegurança entre docentes de enfermagem. Revista de Pesquisa: Cuidado é Fundamental Online, 9(1), 137-143.

Oliveira, J. S., Macedo, M. P., Morais, R. L. G. L., Tanan, M. S., \& Yarid, S. D. (2017). Biossegurança sob a ótica dos graduandos de enfermagem. Rev. enferm. UERJ, 25, e14074-e14074.

Oliveira, K. R. E., \& Braga, E. M. (2016). O desenvolvimento das habilidades comunicativas e a atuação do professor na perspectiva do aluno de enfermagem. Revista da Escola de Enfermagem da USP, 50, 32-38.

Oliveira, L. B., Nunes, B. M. V. T., \& Mourão, L. F. (2015). Demographic profile of the student's undergraduate degree in nursing. Revista de Enfermagem da UFPI, 4(1), 33-9.

Reza, C. G., Ferreira, M. A., Silva, R. C., Gandarilla, J. V., Solano, G. S., \& Martínez, V. G. (2016). Profile of Mexican students in nursing clinics. Escola Anna Nery, 20(1), 11-16.

Santos Junior, E. P., Batista, R. R. A. M., Almeida, A. T. F., \& Abreu, R. A. A. (2015). Acidente de trabalho com material perfurocortante envolvendo profissionais e estudantes da área da saúde em hospital de referência. Revista Brasileira de Medicina do trabalho, 13(2), 69-75.

Santos, P. B., Hermes, D. M., Susin, L., \& Moreira, T. R. (2017). Análise do conhecimento em biossegurança de acadêmicos formandos da área da saúde. Revista Uningá, 53(1), 45-50. 